\section{Metaanalyse: Angiogenesehemmung beim NSCLC}

Der Signalweg des vaskulären endothelialen Wachstumsfaktors ist ein wichtiges Ziel der Krebstherapie. Eine Metaanalyse quantifiziert Wirksamkeit und Sicherheit solcher Therapien beim nichtkleinzelligen Lungenkarzinom (NSCLC).

$V^{2}$ erglichen wurden die gepoolten Ergebnisse zu objektiven Ansprechraten (ORR), Krankheitskontrollraten (DCR), progressionsfreiem (PFS) und Gesamtüberleben (OS) sowie die häufigsten unerwünschten Ereignisse (AEs) aus 33 randomisierte Studien mit insgesamt 7.396 Patienten. 23 Studien verglichen die Tyrosinkinaseinhibitoren (TKI) Vandetanib, Sunitinib, Cediranib, Sorafenib, Pazopanib, Mosetanib und Nintedanib mit nicht antiangiogenetisch wirksamen Therapien, zehn Studien setzten antiangiogene Antikörper wie Bevacizumab, Aflibercept und Ramucirumab ein. 13 der Studien untersuchten den Einsatz in der Erstlinie, 17 in der zweiten und höheren Therapielinien, zwei in der Erhaltungstherapie.

Im Vergleich zu den nicht antiangiogenen Therapien waren die Angiogenesehemmer mit einer signifikanten Verbesserung sowohl des PFS (Hazard Ratio [HR] 0,81; $\mathrm{p}<0,001)$ als auch des OS (HR 0,95; $\mathrm{p}=0,004)$, einer höheren ORR (Relatives Risiko [RR] 1,54; $\mathrm{p}<0,001$ ) und einer verbesserten DCR assoziiert (RR 1,18; $<<0,001)$. Sowohl TKI als auch Antikörper gingen mit signifikant längerem PFS einher, ein knapp signifikant längeres OS fand sich aber nur für die Antikörper-Therapien (HR 0,91; p 0,010). Für die Angiogenesehemmung ergab sich in jeder Linie ein signifikanter Vor- teil für das PFS, aber nur für die Zweitlinie ein signifikant verbessertes OS (HR $0,95 ; \mathrm{p}=0,018)$. Alle Monotherapien verlängerten das PFS, ein Vorteil beim OS war nur für Kombinationen nachweisbar. Mit der Angiogenesehemmung assoziierte AE waren gut vorhersagbar und beherrschbar (Bluthochdruck, Neutropenie, Thrombozytopenie). Bei Nebenwirkungen des Grads 3 und 4 waren noch Blutungen signifikant häufiger unter Angiogenesehemmung.

Fazit: Angiogenesehemmer sind nach dieser Metaanalyse nicht anti-angiogen wirksamen Therapien beim fortgeschrittenen NSCLC überlegen. Wichtig wäre die Identifizierung prädiktiver Biomarker, um Patienten zu identifizieren, die besonders profitieren. Friederike Klein

Hong S et al. Efficacy and safety of angiogenesis inhibitors in advanced non-small cell lung cancer: a systematic review and meta-analysis. J Cancer Res Clin Oncol. 2015;141(5):909-21.

\title{
Kleiner, aber signifikanter Lebenszeitgewinn beim NSCLC
}

Bei Patienten mit einem plattenepithelialen nichtkleinzelligen Lungenkarzinom (NSCLC) sind die Therapieoptionen bisher begrenzt. Afatinib wurde in dieser Situation im Vergleich zu Erlotinib geprüft.

A n der offenen Phase-III-Studie LUXLung 8 nahmen weltweit 795 Patienten mit einem Plattenepithelkarzinom des Stadiums IIIb oder IV teil, die nach mindestens vier Zyklen einer platinbasierten Chemotherapie progredient waren. Randomisiert erhielten sie entweder
Afatinib, den irreversiblen Inhibitor der ErbB-Rezeptorfamilie (40 mg/d), oder den EGFR-Inhibitor Erlotinib (150 $\mathrm{mg} / \mathrm{d}$ ) bis zum Progress. Der primäre Endpunkt - das progressionsfreie Überleben (PFS) - zeigte nach median 6,7 Monaten einen signifikanten Vorteil für
Tab. 1: Therapieansprechen unter Afatinib und Erlotinib (Auswahl)

\begin{tabular}{|l|l|l|l|}
\hline & Afatinib $(n=398)$ & Erlotinib $(n=397)$ & $p$-Wert \\
\hline $\begin{array}{l}\text { Krankheitskontrolle } \\
\text { - ohne Patienten mit NN }\end{array}$ & $201(51 \%)$ & $157(40 \%)$ & 0,0020 \\
\hline $\begin{array}{l}\text { objektives Ansprechen } \\
\text { - CR }\end{array}$ & $22(6 \%)$ & $114(29 \%)$ & 0,0170 \\
\hline - PR & $1(<1 \%)$ & $11(3 \%)$ & 0,0551 \\
\hline Stabile Erkrankung & $21(5 \%)$ & 0 & \\
\hline
\end{tabular}

$N N=$ nicht $C R$ und nicht $P D ; C R=$ komplettes $A n s p r e c h e n ; P R=$ partielles Ansprechen
Afatinib mit im Median 2,4 gegenüber 1,9 Monaten unter Erlotinib (Hazard Ratio [HR] 0,82; $\mathrm{p}=0,0427)$. Die Primäranalyse des Gesamtüberlebens (OS) nach median 18,2 Monaten zeigte ebenfalls einen zwar kleinen, aber signifikanten Vorsprung der Afatinib-Gruppe mit median 7,9 gegenüber 6,8 Monaten (HR $0,81 ; p=0,0077)$. Auch zu diesem Zeitpunkt war das mediane PFS unter Afatinib besser als unter Erlotinib (2,6 vs. 1,9 Monate; HR 0,81; p = 0,0103) - bei vergleichbaren Nebenwirkungsprofilen.

Fazit: Aufgrund der signifikanten, wenn auch geringen Verlängerung von PFS und OS durch Afatinib und der beherrschbaren Nebenwirkungen sowie der oralen Gabe bezeichnen die Autoren Afatinib als mögliche zusätzliche Option zur Behandlung des Plattenepithelkarzinoms der Lunge. Friederike Klein

Soria JC et al. Afatinib versus erlotinib as second-line treatment of patients with advanced squamous cell carcinoma of the lung (LUX-Lung 8): an open-label randomised controlled phase 3 trial. Lancet Oncol. 2015;16(8):897-907. 